\title{
Characteristics of the ALICE Silicon Drift Detector.
}

\author{
A. Rashevsky b,1, V. Bonvicini ${ }^{\mathrm{b}}, \mathrm{P}$. Burger ${ }^{\mathrm{c}}, \mathrm{P}$. Cerello $^{\mathrm{a}}$, \\ E. Crescio ${ }^{a}$, P. Giubellino ${ }^{\text {a }}$, R. Hernández-Montoya ${ }^{\mathrm{a}, 2}$, \\ A. Kolojvari ${ }^{a, 3}$, L.M. Montaño ${ }^{\mathrm{a}, 2}$, D. Nouais $^{\mathrm{a}}$, C. Piemonte $^{\mathrm{b}}$, \\ F. Tosello ${ }^{\mathrm{a}}$, A. Vacchi ${ }^{\mathrm{b}}$, R. Wheadon ${ }^{\mathrm{a}}$, \\ for the ALICE Collaboration \\ ${ }^{a}$ INFN Sezione di Torino, Italy \\ ${ }^{\mathrm{b}}$ INFN Sezione di Trieste, Italy \\ ${ }^{\mathrm{c}}$ Canberra Semiconductor, $N$ V 2250 Olen, Belgium
}

\begin{abstract}
A Silicon Drift Detector (SDD) with an active area of $7.0 \times 7.5 \mathrm{~cm}^{2}$ has been designed, produced and tested for the ALICE Inner Tracking System. The development of the SDD has been focused on the capability of the detector to work without an external support to the integrated high voltage divider. Several features have been implemented in the design in order to increase the robustness and the long term electrical stability of the detector. One of the prototypes has been tested in a pion beam at the CERN SPS. Preliminary results on the position resolution are given.
\end{abstract}

\section{Description of the detector.}

The Inner Tracking System (ITS) of the ALICE experiment at LHC will consist of six cylindrical layers of high precision position-sensitive detectors. 260 silicon drift detectors (SDDs) will equip the 3rd and the 4th layer, providing position information in two dimensions and dE sample $[1,2]$. Few detectors of the most recent design, realized by the end of 1999 and called ALICED1B, have been produced on 5-inch, diameter neutron transmutation doped

$\overline{1}$ Corresponding author. E-mail: rashevsky@ts.infn.it

2 also at CINVESTAV Mexico City, Mexico

3 also at St. Petersburg University, St. Petersburg, Russia 
(NTD), silicon wafers with a resistivity of $3 \mathrm{k} \Omega \mathrm{cm}$ and a thickness of $300 \mu \mathrm{m}$. A dedicated study [3] demonstrated that NTD silicon was suitable for the production of SDDs, providing doping fluctuations below $2 \%$ (r.m.s.) with maximum variations of about $6 \%$. The detector has a bi-directional structure, where electrons drift from the central $p^{+}$cathode towards two linear arrays of $n^{+}$anodes. It has a hexagonal shape (fig. 1) with a rectangular sensitive

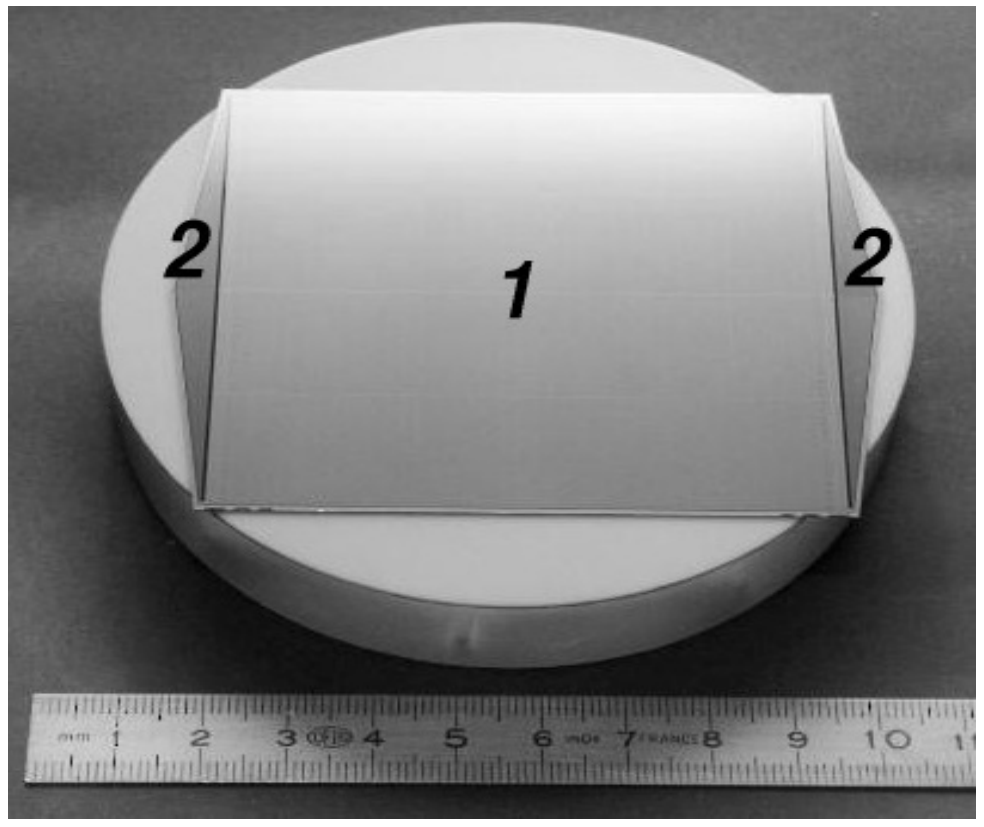

Fig. 1. Picture showing a SDD of the design ALICE-D1B. The numbers correspond to: (1) sensitive area; (2) guard area.

area that allows to minimise the overlapping of adjacent SDDs in the ladder assembly. For each half-detector there are 292 drift cathodes with a pitch of $120 \mu \mathrm{m}$ and 256 anodes with a pitch of $294 \mu \mathrm{m}$. So, the length of the sensitive area delimited by two arrays of anodes is $70.0 \mathrm{~mm}$, while the width of the sensitive area along the anodes is $75.3 \mathrm{~mm}$. The sensitive-to-total-area ratio is $88 \%$. At the flanks there are two triangular areas constituted by the guard $p^{+}$cathodes. There is one guard cathode every two drift cathodes, so the potential difference between adjacent guards is twice that between adjacent drift cathodes. The detector is planned to work at a potential difference of about $8 \mathrm{~V}$ between adjacent drift cathodes which corresponds to a drift field of $670 \mathrm{~V} / \mathrm{cm}$. The pitch of the guard cathodes is $32 \mu \mathrm{m}$, resulting in a drift field in the guard areas of $5000 \mathrm{~V} / \mathrm{cm}$ making them one of the most critical structures of a silicon drift detector. The detector is biased through a set of integrated high-voltage dividers made of high resistivity $p^{+}$implants. There are separate dividers to bias independently the drift cathodes of each halfdetector, and the guard cathodes (fig. 2). In this way, possible distortions of the potential distribution on the drift cathodes of one half-detector, or on the guard cathodes, will not be directly transferred to the other one. The value of 


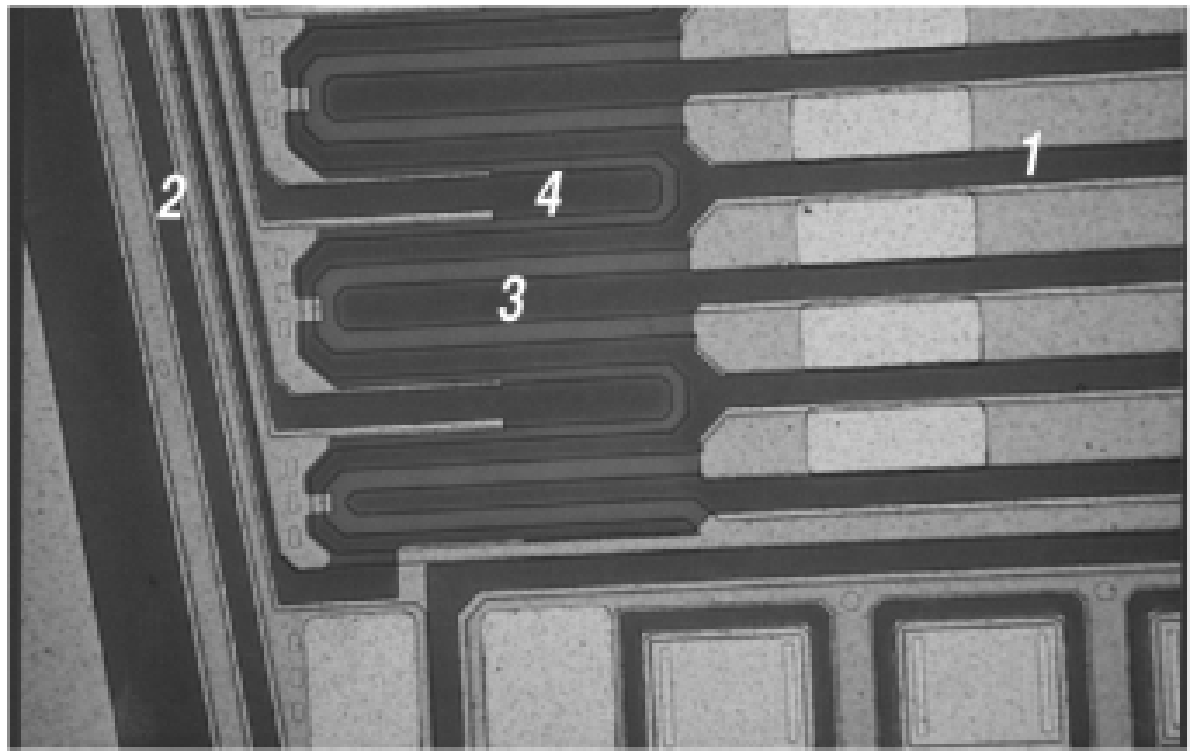

Fig. 2. Details of the H.V. integrated divider region: (1) drift cathode; (2) guard cathode; (3) divider resistor for the drift cathodes; (4) divider resistor for the guard cathodes.

one integrated resistor connecting adjacent drift cathodes is $170 \mathrm{k} \Omega$.

Designing a SDD without an external divider, one of the keystones is to ensure its long-term electrical stability. It is known, that the leakage and punchthrough currents flowing in a silicon detector can be very dependent on the environmental conditions, if one does not foresee special solutions in the detector design to attenuate this influence [4,5]. From this point of view, in case of the SDD, the punch-through phenomenon is the most critical. When the voltage difference between adjacent $p^{+}$cathodes reaches a critical value $U_{p t}$, a hole current starts to flow. This phenomenon can be considered as a parasitic resistor inserted in parallel to the implanted resistor of the divider, altering, thus, the linear potential distribution on the cathodes. In the ideal situation, i.e. without considering the environmental conditions, the value $U_{p t}$ depends on the positive fixed oxide charge, the silicon resistivity, and on the distance between adjacent cathodes. Actually, negative charges, coming from the environment, are deposited on the outer oxide surface and compensate progressively in time the positive charges inside the oxide. The dynamics of the negative charge accumulation on the outer oxide surface, the amount of this charge and its compensating capability depend on different factors: 1) whether the detector is biased or not; 2) the level of the environmental humidity; 3 ) whether the adjacent cathodes have the metallisation above $p^{+}$implants, and, how much this metallisation overhangs the oxide to form a field-plate; 4) the oxide thickness; 5) the time during which the detector is biased. Under certain 
conditions this negative charge does not only partially or completely compensates the positive charge inside the oxide, but starts to prevail and causes the formation of an inversion layer under the oxide. Consequently, the value $U_{p t}$ can decrease in a dramatic way, and the seemingly perfect linearity of the potential distribution on the drift cathodes, measured a short time after having biased the SDD, becomes altered on a longer time scale. This decrease of $U_{p t}$ can take hours and even days before reaching an asymptotic limit. To overcome this problem we implemented for the first time in the design ALICE-D1B a cathode metallisation with 'punch-through safe' field-plates. Figure 3 gives
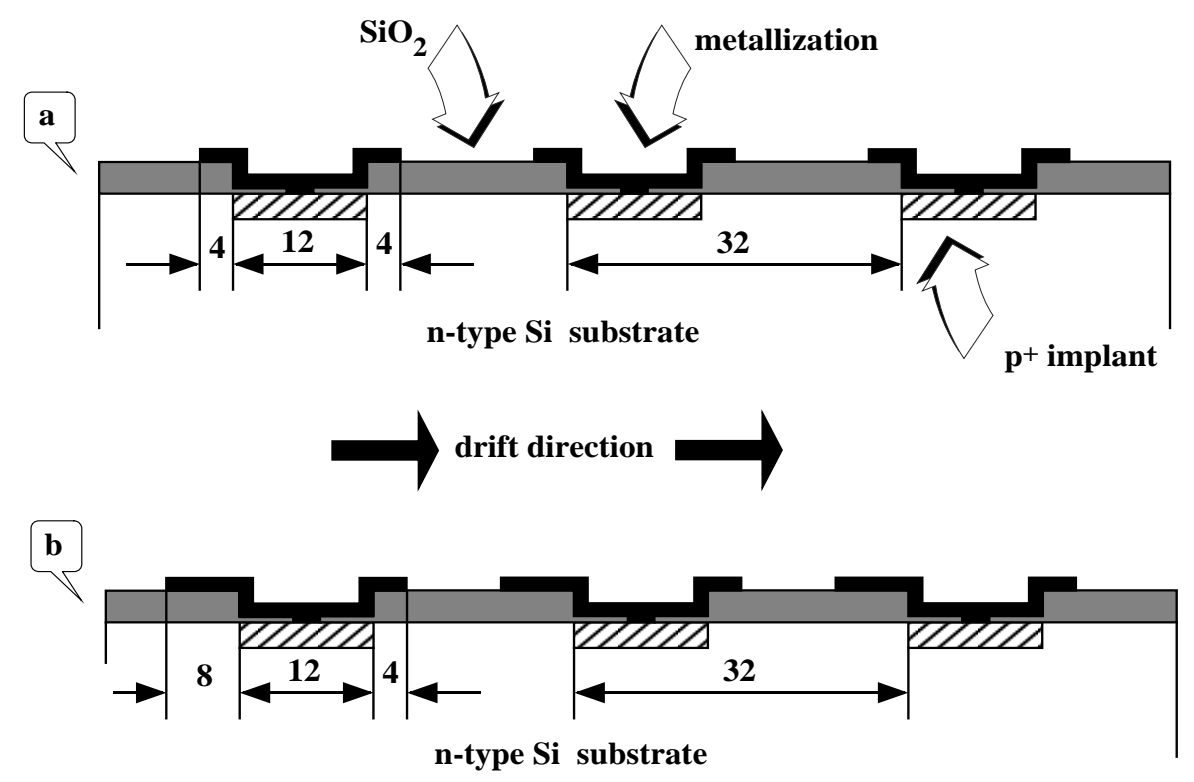

Fig. 3. Geometry of the guard structure implemented in the actual design ALICE-D1B (b), and in the precedent design ALICE-D1 (a).

a comparison between the old guard structure, where the metal overhang is $4 \mu \mathrm{m}$ at both edges of the $p^{+}$implant, forming a symmetrical field-plate, and the new one, where the 'inward' field-plate is $8 \mu \mathrm{m}$ wide. This inhibits the compensation at the edge of the more positive implant, increasing effectively the asymptotic limit for $U_{p t}$. Figure 4 presents the value $U_{p t}$ measured for the two guard structures shown in fig. 3 as a function of the time during which the bias voltages are applied. The oxide thickness and the positive fixed oxide charge are the same for both structures.

We carefully simulated the collection zone in order to reduce as much as possible the number of individually biased drift cathodes which serve to bring the drifting charge from the middle plane of the detector bulk towards the collecting $n^{+}$anodes. The idea is to keep the drifting charges in the middle plane till the last $150 \mu \mathrm{m}$ from the anodes and then to bring them rapidly to 


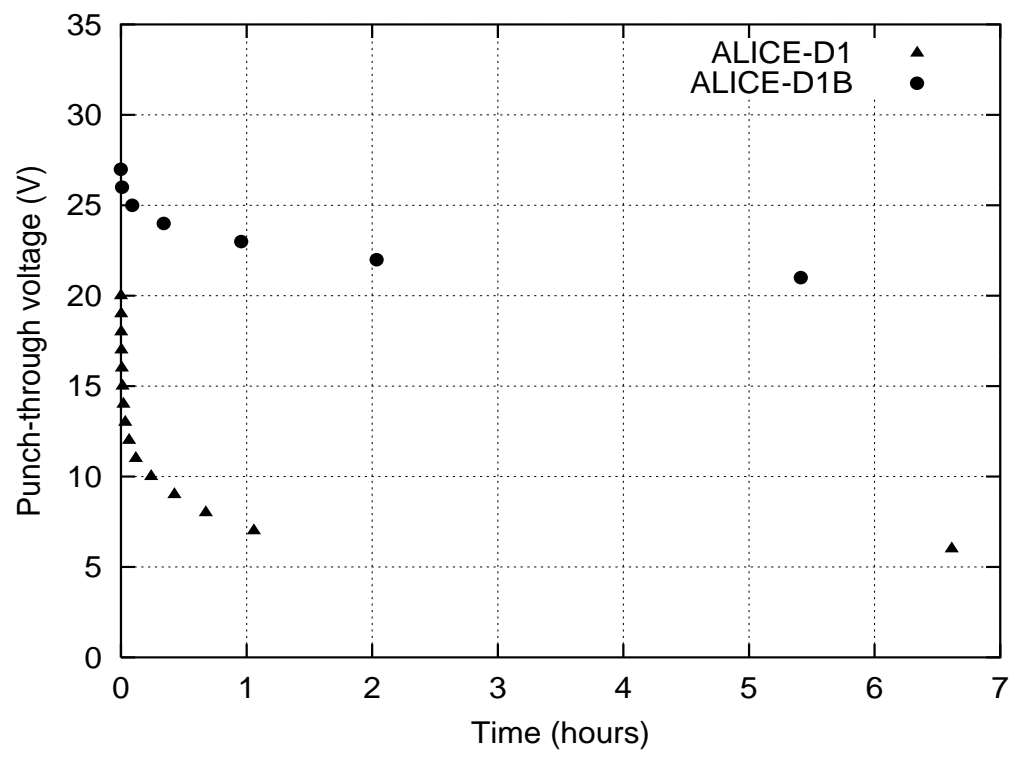

Fig. 4. The value $U_{p t}$ measured for the guard structures of the design ALICE-D1B and ALICE-D1 (see fig. 3) as a function of the time during which the bias voltages are applied. The asymptotic limit for $U_{p t}$ is much higher in presence of the enlarged 'inward' field-plate (ALICE-D1B).

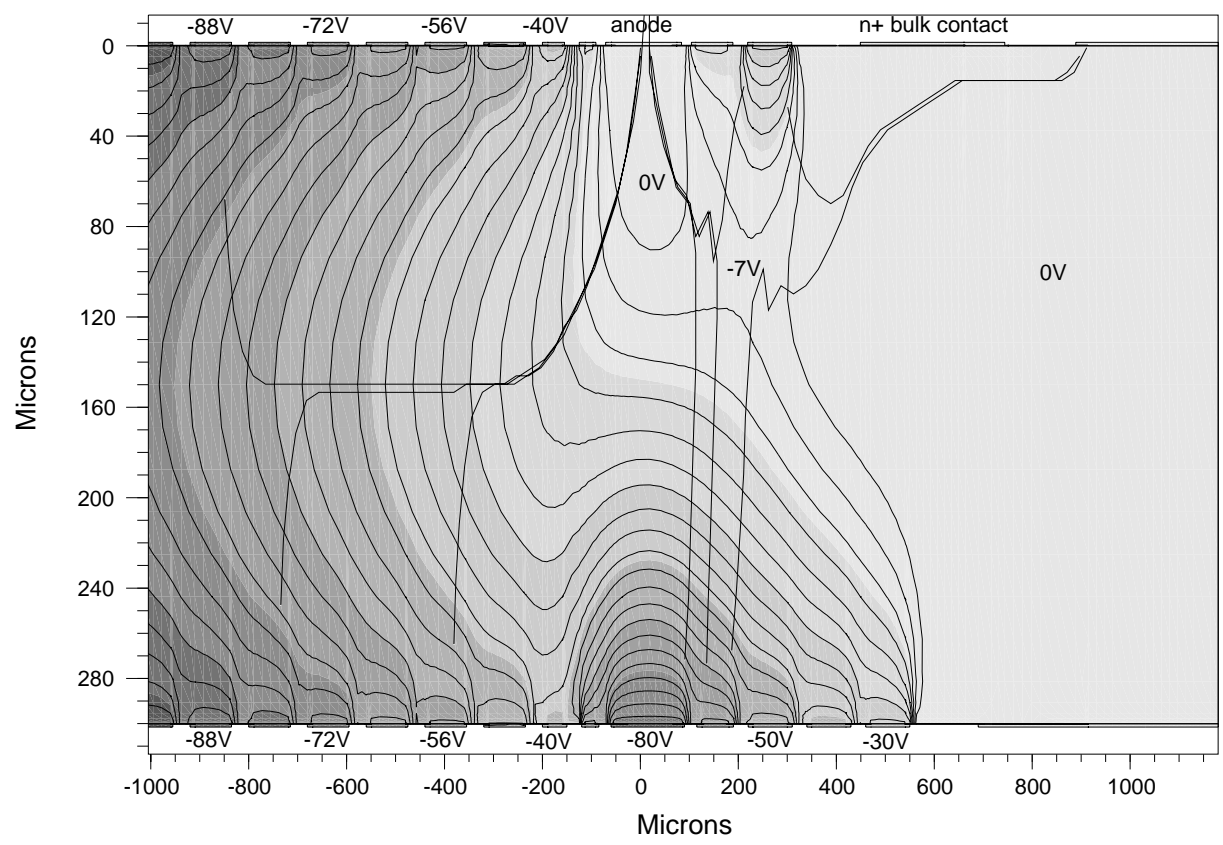

Fig. 5. Simulation of the collection zone. The equipotential map is shown together with trajectories of several electrons 'generated' at different positions. 
the anodes using few $p^{+}$cathodes opposite to the anodes (fig. 5). Thus, the drift field is maintained constant over the whole drift length (except for the last $100 \mu \mathrm{m}$ ), the drift trajectory is safely distant from the anode side and the drifting charge cannot be trapped by the potential 'pockets' near the $\mathrm{Si}-\mathrm{SiO}_{2}$ interface. Relying on simulation results, we tried this biasing scheme of the collection zone in the SDD prototypes, and it proved to be valid $[6,7]$. Therefore we introduced in the actual design the minimised collection zone which consists only of few cathodes biased independently from the integrated high voltage divider. The 'grid' cathode on the anode side separates anodes one from another, while two 'kick up' cathodes on the opposite side force the drifting charge towards the anodes array. Four outermost cathodes (one on the anode side and three on the opposite side) serve to ensure electrical separation of anodes from the $n^{+}$perimeter.

To calibrate the drift time for the temperature variations across the sensitive area, we implemented 'point-like' MOS charge injectors, which proved to be a very useful and reliable structure $[6,8,9]$. In each half-detector there are two arrays of injectors at distances of $17.6 \mathrm{~mm}$ and $35.0 \mathrm{~mm}$ from the anodes. The injector pitch is $2.35 \mathrm{~mm}$ (every eight anodes).

\section{Laboratory tests.}

Here is a brief description of the tests that each SDD coming from the mass production will have to undergo in order to verify whether this detector satisfies the acceptance conditions imposed by the experiment. First, it is important to make, in a simple and fast way, a preliminary (I-V) measurement in order to perform a first selection. Since all drift cathodes of one side are connected together through the integrated voltage divider, it is enough to use two probes to bias all cathodes of this side. This is to be done for both sides of the detector. We require that the leakage current measured in this way should not be higher than few $\mu A$ at a bias voltage of $-30 \mathrm{~V}[10]$.

Regarding the anode leakage current, we impose an upper limit of $100 \mathrm{nA}$ for the current per anode at a bias voltage of $-2400 \mathrm{~V}$. This requirement is related to the design of the front-end electronics $[2,10]$. In addition, it is allowed to have at most $2 \%$ of the anodes with a current between 100 and $500 n A$. Figure 6 shows the distribution of the anode current for both the halves of one of the tested SDDs, biased at $-2400 \mathrm{~V}$.

Another important characteristics of a SDD is the linearity of the potential distribution on the drift cathodes, which is mandatory to maintain the drift field constant (for an easy reconstruction of the impact coordinate along the drift). Both, the hole component of the leakage current entering the cathode 


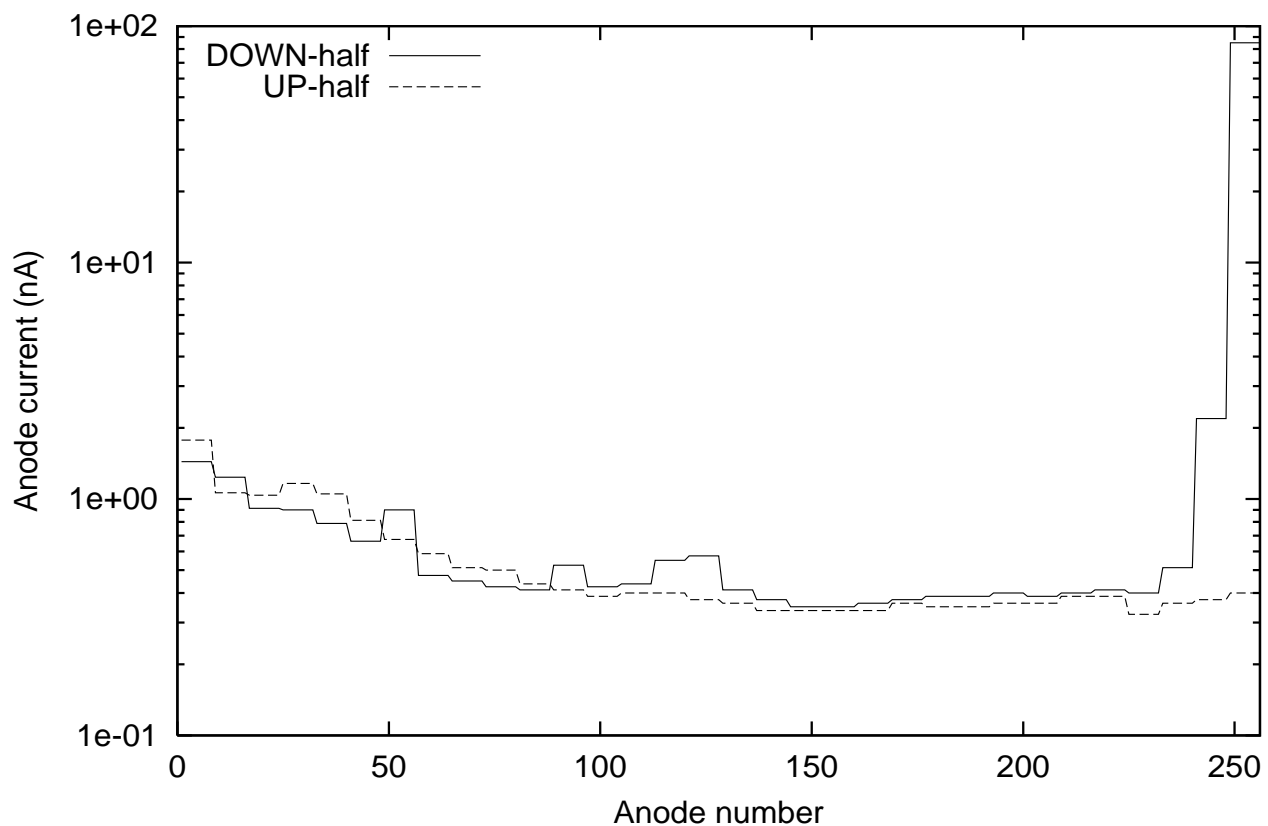

Fig. 6. Distribution of the anode current measured for the two halves of the SDD. The current was measured connecting together 8 anodes at a time and the plot presents the resulting average current per anode.

chain and the eventual punch-through current between cathodes, can distort this linearity. Measuring the potential drop between consecutive drift cathodes allows to understand the nature of the current (punch-through or leakage) that enters the cathode chain in addition to the integrated divider current. Moreover, from the slope of the potential drop plotted as a function of the drift cathode number it is possible to extract the hole leakage current entering one cathode [10]. We require that, under the working bias conditions corresponding to a drift field of $670 \mathrm{~V} / \mathrm{cm}$, there is no punch-through between neither guard nor drift cathodes, while the average hole leakage current entering one cathode should not be greater than $40 \mathrm{nA}$ (it corresponds to an upper limit of $100 \mathrm{nA}$ for the current per anode). Figure 7 shows the potential drop between ten consecutive drift cathodes as a function of the drift cathode number, measured on both sides of one half-detector.

\section{Test beam results on the SDD position resolution.}

One of the ALICE-D1 SDDs was tested on a pion beam at the CERN SPS accelerator. The only difference between the designs ALICE-D1 and ALICED1B is the 'punch-through safe' cathode metallisation. The beam momentum was $345 \mathrm{GeV} / \mathrm{c}$. As a reference we used a telescope made of single-sided silicon microstrip detectors with a strip pitch of $50 \mu \mathrm{m}$, which gave a precision of 
SDD \#38995 (AL1b-2)

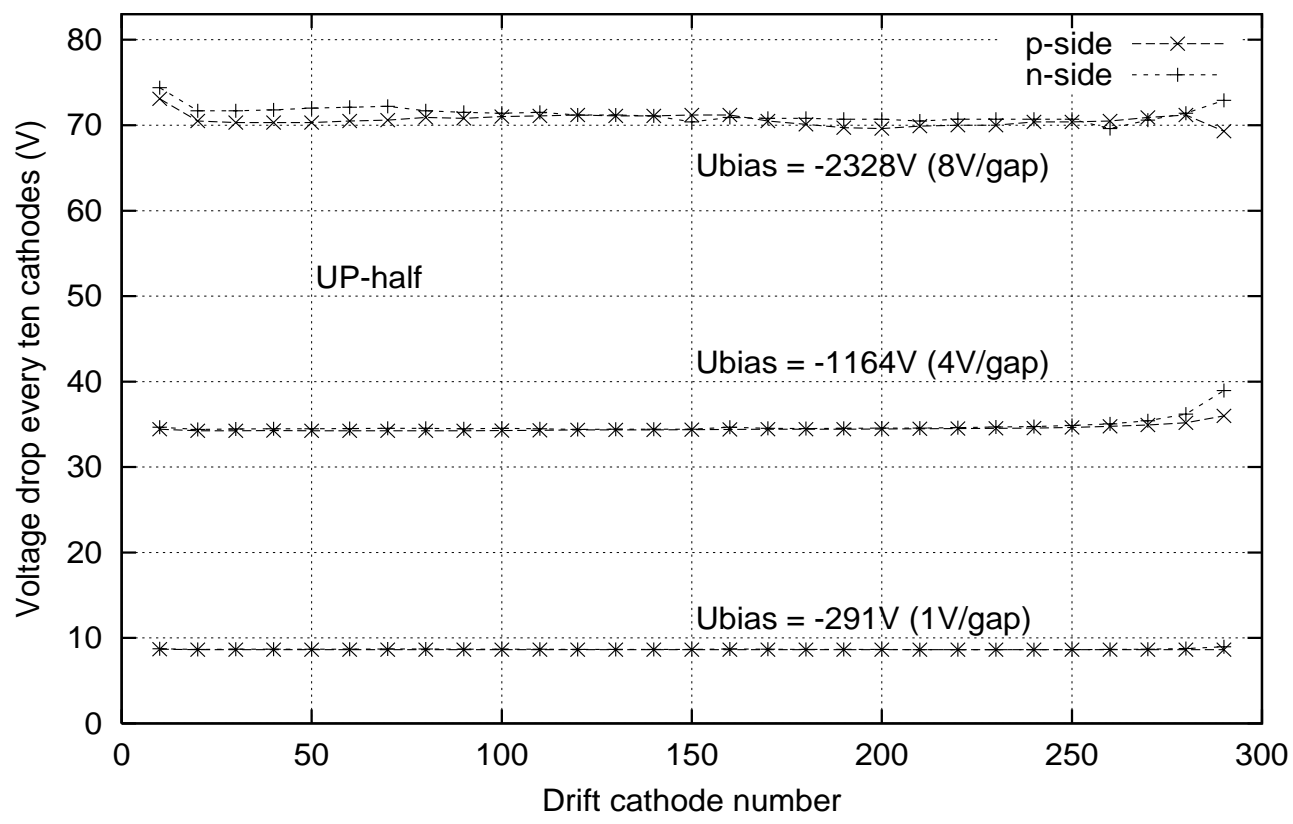

Fig. 7. Potential drop between ten consecutive drift cathodes as a function of the drift cathode number, measured on both sides of one half-detector for three different bias voltages.

$5 \mu m$ r.m.s. in the determination of the particle impact point in the SDD plane [2]. The position resolution along the anode direction versus drift distance is presented in fig. 8. The data were taken at a drift field of $583 \mathrm{~V} / \mathrm{cm}$ and the analysis was done for a fraction of the drift area $19.0 \mathrm{~mm}$ wide (64 anodes). The position resolution curve was obtained in the following way [11]. First,

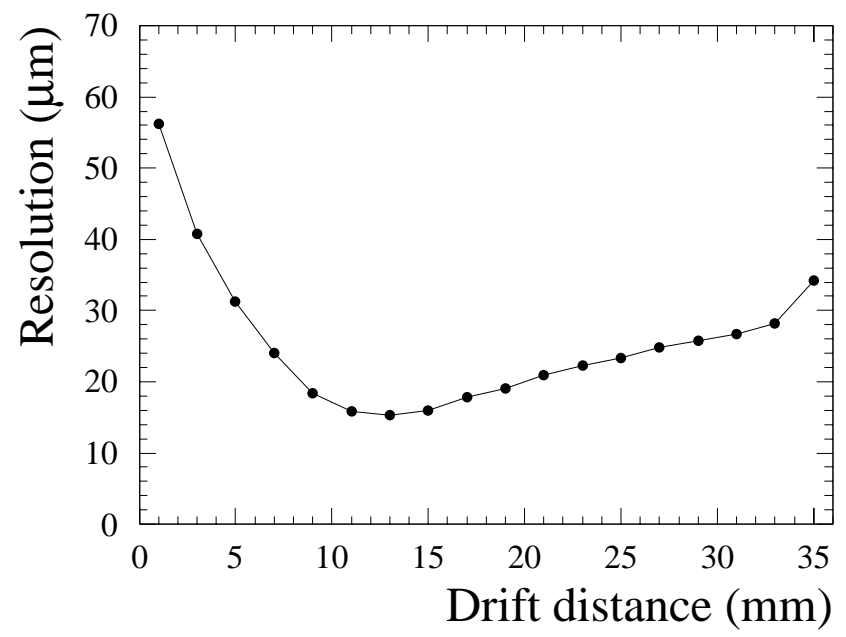

Fig. 8. Position resolution along the anode direction applying correction of the systematic deviations.

it was evaluated as a difference between the cluster position measured by the SDD and the impact point projected by the microstrip telescope. Then, 
the drift area under analysis was mapped to search for systematic deviations created by non-uniformities of the dopant concentration in the detector bulk. The introduction of a correction for the above systematic effects results in a resolution curve shown in fig. 8. For what concerns the position resolution along the drift direction, an elaborated analysis is still under development. Presently, the value averaged over all the drift distances is $35 \mu \mathrm{m}$.

R. H. wishes to thank Conacyt from Mexico and ICTP from Trieste. L. M. M. wishes to thank Conacyt for financial support.

\section{References}

[1] ALICE Collaboration, CERN/LHCC 95-71.

[2] ALICE Collaboration, CERN/LHCC, 99/12.

[3] S. Beole' et al., to be published in Nucl. Instr. and Meth.

[4] A. Longoni et al., Nucl. Instr. and Meth. A288 (1990) 35-43.

[5] A. Bischoff et al., Nucl. Instr. and Meth. A326 (1993) 27-37.

[6] V. Bonvicini et al., Nucl. Instr. and Meth. A439 (2000) 476.

[7] D. Nouais et al., Nucl. Phys. B (Proc. Suppl.) 78 (1999) 252-258.

[8] V. Bonvicini et al., Il Nuovo Cimento, Vol. 112A N. 1-2, January-February 1999, 137-146.

[9] D. Nouais et al., Nucl. Instr. and Meth. A (in press)

[10] C. Piemonte, A. Rashevsky, D. Nouais, INFN/TC - 00/04

[11] D. Nouais et al., in These Proceedings. 